\title{
Singlet Oxygen-Responsive Polymeric Nanomedicine for Light-Controlled Drug Release and Image-Guided Photodynamic-Chemo Combination Therapy
}

De-Chao Yang, ${ }^{\dagger}$ Shuai Wang, ${ }^{\dagger}$ Xiao-Lu Weng, ${ }^{\dagger}$ Hong-Xia Zhang, ${ }^{\dagger}$ Jian-Yong Liu, ${ }^{*, \dagger}, \ddagger, \S$ and Zhonghui Lin*,

†National \& Local Joint Biomedical Engineering Research Center on Photodynamic Technologies, College of Chemistry, Fuzhou University, Fuzhou 350108, China

\$Key Laboratory of Molecule Synthesis and Function Discovery, Fujian Province University, College of Chemistry, Fuzhou University, Fuzhou 350108, China

\$State Key Laboratory of Photocatalysis on Energy and Environment, College of Chemistry, Fuzhou University, Fuzhou 350108, China

Corresponding Author.

E-mail: 1kw82@fzu.edu.cn (Jian-Yong Liu); zhonghui.lin@fzu.edu.cn (Zhonghui Lin) 


\section{Table of Contents}

\section{Materials}

Figure S1. Structures of BDP and PTX, and synthesis of SOR-mPEG- $b$-PCL and mPEG- $b$-PCL

Figure S2. $\quad{ }^{1} \mathrm{H}$ NMR spectrum of polymer 1

Figure S3. $\quad{ }^{1} \mathrm{H}$ NMR spectrum of polymer 2

Figure S4. $\quad{ }^{1} \mathrm{H}$ NMR spectrum of SOR-mPEG- $b$-PCL

Figure S5. $\quad{ }^{1} \mathrm{H}$ NMR spectrum of $\mathrm{mPEG}-b$-PCL

Figure S6. Infrared spectra of mPEG, 1, 2, SOR-mPEG- $b$-PCL, mPEG- $b$-PCL

Table S1. Characteristics of polymers

Figure S7. Critical micelle concentration of mPEG- $b$-PCL

Figure S8. $\quad$ Particle size distribution and TEM images of C-NP and C-NP $\mathrm{BDP}_{\mathrm{BDTX}}$

Table S2 Physicochemical characteristics of BDP/PTX loaded nanoparticles

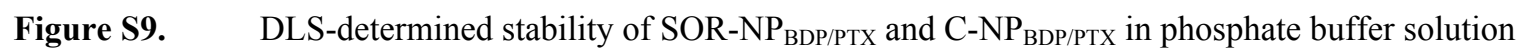

Figure S10. Standard curve line of BDP and PTX

Figure S11. The fluorescence emission spectra of DMA after different durations of $660 \mathrm{~nm}$ laser

Figure S12 GPC profiles of mPEG- $b$-PCL with BDP $(\sim 5 \mathrm{wt} \%)$ with or without laser irradiation

Figure S13. Average size change and TEM images of SOR-NP under various irradiation time

Figure S14 Cumulative PTX release profiles from C-NP $\mathrm{BDP}_{\mathrm{PTX}}$ without or with light irradiation

Figure S15 Cellular uptakes in HepG2 cells

Figure S16 Cellular uptakes in MCF-7 cells

Figure S17 Intracellular ${ }^{1} \mathrm{O}_{2}$ production in MCF-7 cells using DCFH-DA assay

Figure S18 Dark- and photo-cytotoxicity of SOR-NP and C-NP against HepG2 and MCF-7.

Figure S19 Fluorescence live cell images in MCF-7

Figure S20 H\&E staining analysis of tumor tissues

Figure S21 H\&E staining of heart, liver, spleen and kidney 


\section{Materials}

Monomethoxy poly(ethylene glycol) (mPEG-OH, $\mathrm{M}_{\mathrm{n}}=2000 \mathrm{~g} \mathrm{~mol}^{-1}$ ), propiolic acid (98\%), $\quad p$-toluenesulfonic acid $\quad(98 \%), \square \varepsilon$-caprolactone $\quad(99 \%)$, 4-piperidinemethanol (98\%), stannous octoate $\left(\mathrm{Sn}(\mathrm{Oct})_{2}, 98 \%\right)$, paclitaxel (PTX, 97\%), and 9, 10-dimethylanthracene (DMA, 98\%) were purchased from Energy Chemical Technology Co., Ltd., Shanghai, China. All solvents were obtained from Titan Scientific Co., Ltd., Shanghai, China. Toluene and tetrahydrofuran (THF) were distilled from sodium. MCF-7 human breast cancer and HepG2 human liver cancer cells were obtained from the cell bank of Shanghai Institutes for Biological Sciences. Dulbecco's Modified Eagle Medium (DMEM), fetal bovine serum (FBS), penicillin-streptomycin, and trypsin were purchased from Thermo Fisher Scientific (China) Co., Ltd., Shanghai, China. Confocal dish and 96-well sterile cell culture plate were purchased from NEST Biotechnology Co., Ltd., Wuxi, Jiangsu, China.

DAPI, Lyso-Tracker Red, 2',7'-dichlorofluorescein diacetate (DCFH-DA), 3-(4,5-dimethylthiazol-2-yl)-2,5-diphenyltetrazolium bromide (MTT), and calcein AM were obtained from Dingguo Biotechnology Co., Ltd., Haimen, China.

${ }^{1} \mathrm{H}$ NMR spectra were conducted on a AVANCE III $\left({ }^{1} \mathrm{H}, 400 \mathrm{MHz}\right)$ instrument (Bruker, Karlstuhe, Germany) in $\mathrm{CDCl}_{3}$. Gel permeation chromatography were recorded on Wasters 1515 series equipped with a Waters 2414 refractive-index detector (Milford, USA). High-performance liquid chromatography (HPLC) was performed using C18 $4.6 \times 200 \mathrm{~mm}^{2}$ columns (YL9111S LC, Korea). Nanoparticle size was measured on a Zetasizer NanoZS dynamic light scattering instrument (Malvern Instruments, Worcestershire, U.K.) and transmission electron microscope (JEM-2100F, JEOL, Japan). Electronic absorption and Fluorescence emission spectra were performed on the PerkinElmer Lambda $365 \mathrm{UV}-$ Visible absorption spectrometer (Massachusetts, USA) and VARIAN Carye Elipse Fluorescence Spectrometer (California, USA), respectively. Infrared spectra were conducted on ThermoFisher fourier transform infrared spectrometer (Massachusetts, USA). Cellular uptake and intracellular ROS generation were carried on Olympus FV1000 confocal laser scanning microscope (Tokyo, Japan). In vivo fluorescence imaging was recorded on 
FMTTM 2500LX Fluorescence Molecular Tomography (PerkinElmer, Waltham, MA).

MCF-7 and HepG2 cells were cultured in DMEM medium containing 10\% FBS and $1 \%$ penicillin-streptomycin at $37{ }^{\circ} \mathrm{C}$ in a humidified incubator with a $5 \% \mathrm{CO}_{2}$ atmosphere. Female KunMing mice (5-weeks-old, $\sim 20 \mathrm{~g}$ ) were purchased from the Wushi Laboratory Animal (Fuzhou, China). All animal experiments were approved by the Animal Ethics Committee of Fuzhou University.

(a)

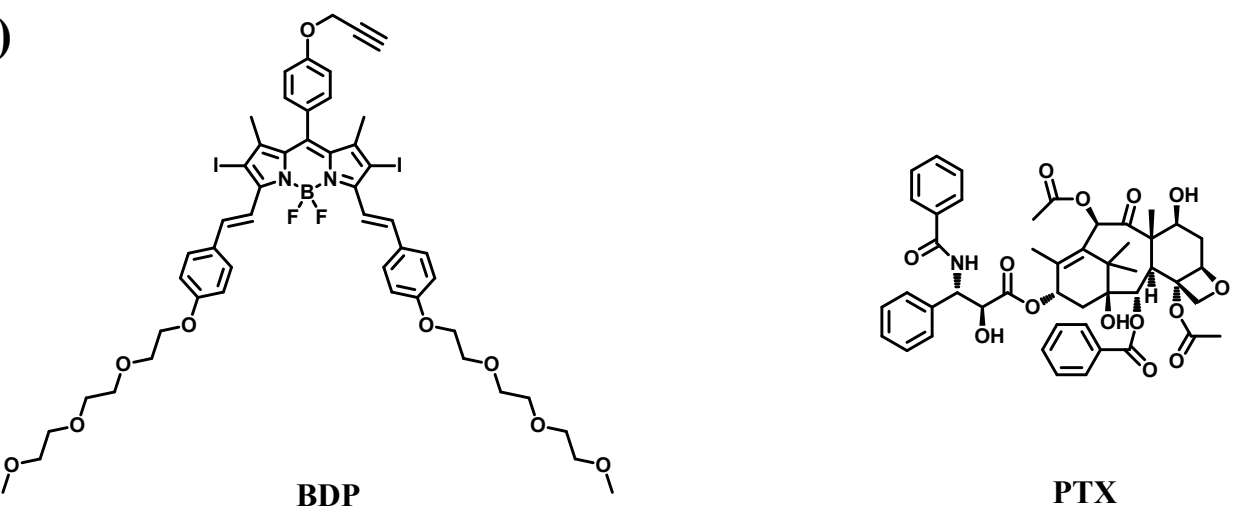

(b)
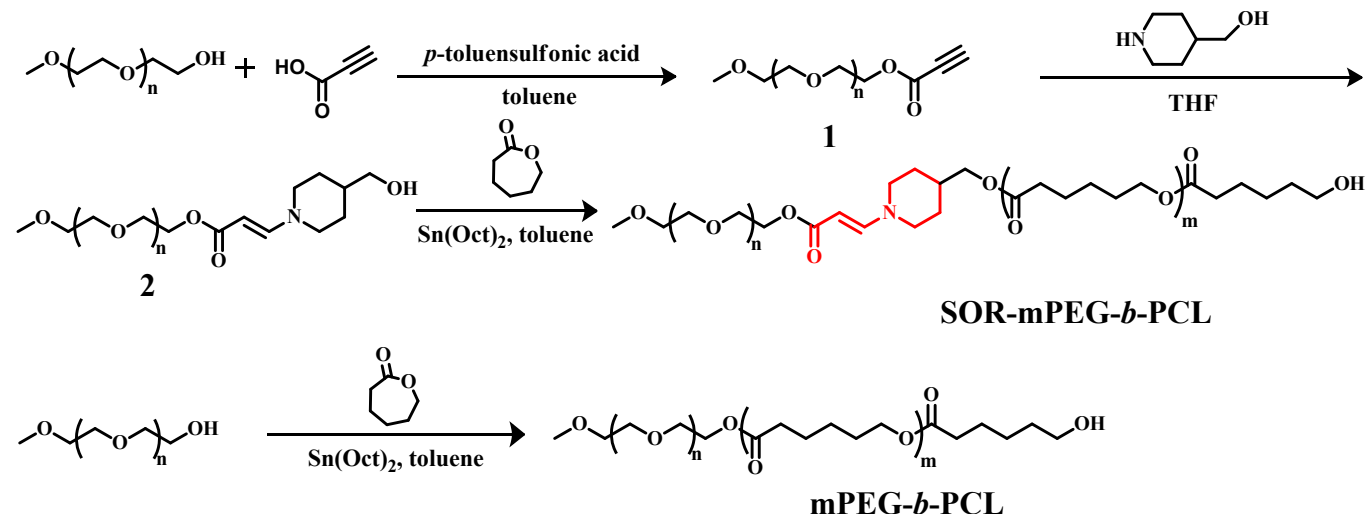

Figure S1. (a) Structures of BDP and PTX; (b) synthesis of the ${ }^{1} \mathrm{O}_{2}$-responsive copolymer SOR-mPEG- $b$-PCL and the control mPEG- $b$-PCL. 


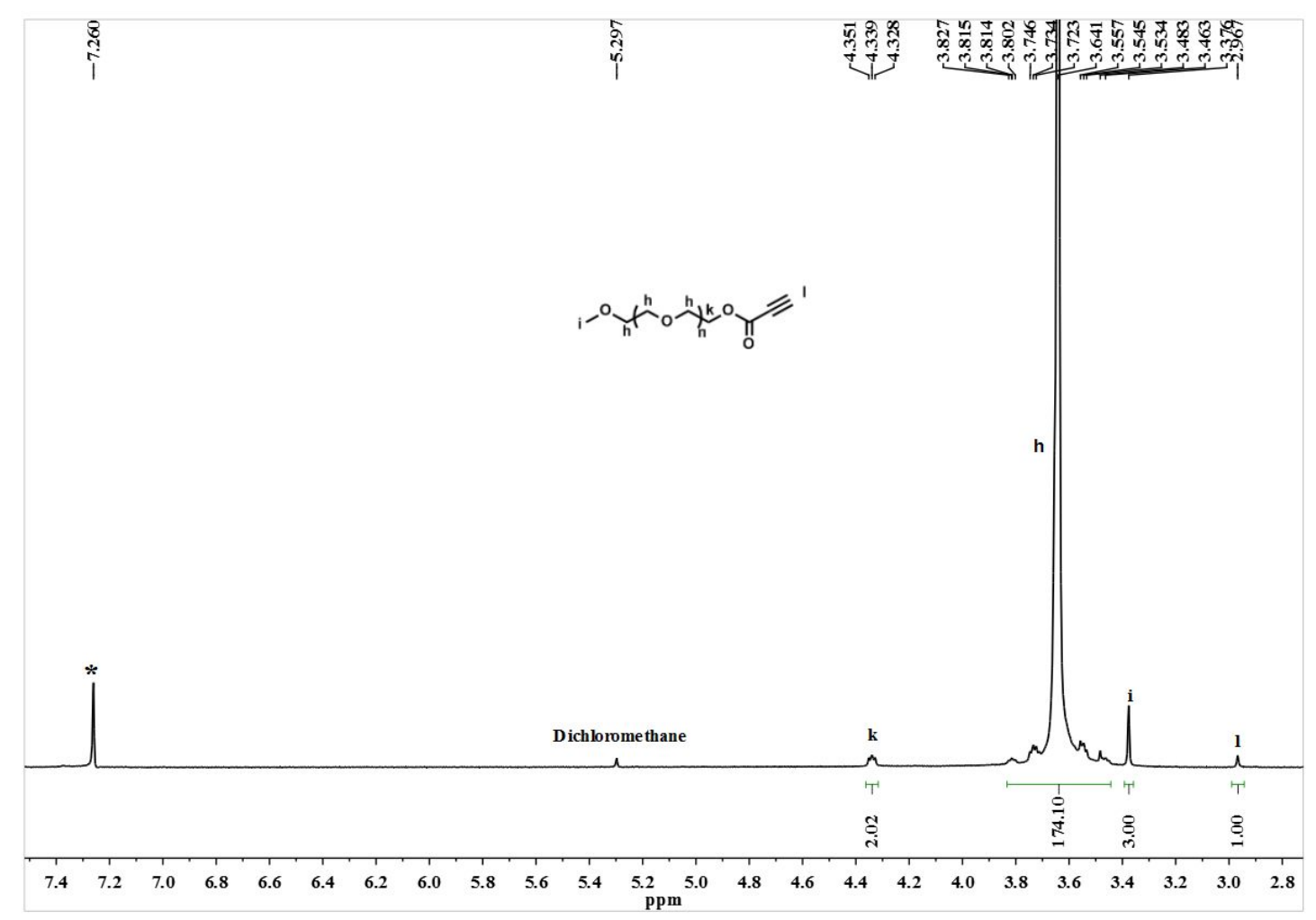

Figure S2. The ${ }^{1} \mathrm{H}$ NMR spectrum of polymer 1 in $\mathrm{CDCl}_{3}$.

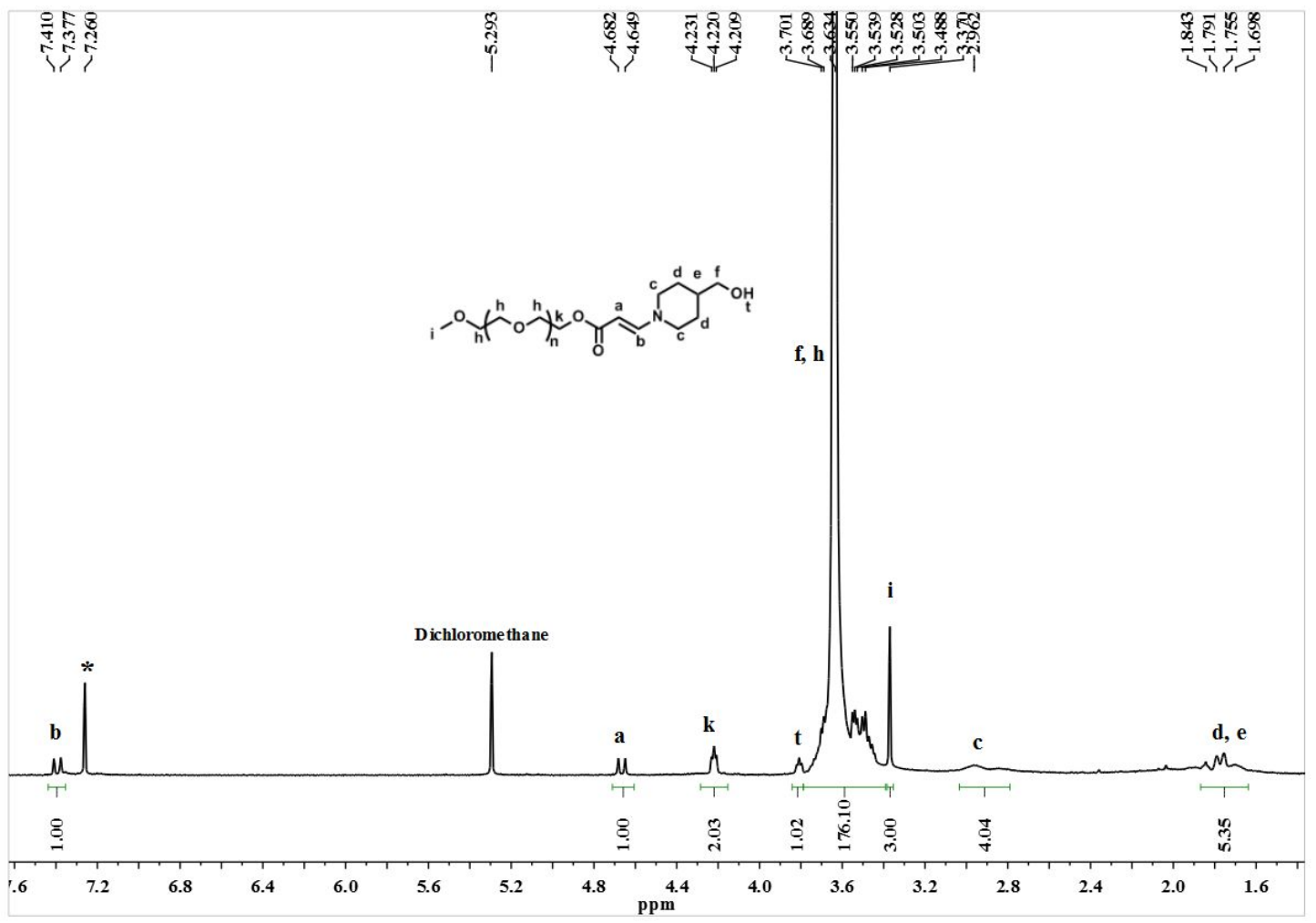

Figure S3. The ${ }^{1} \mathrm{H}$ NMR spectrum of polymer 2 in $\mathrm{CDCl}_{3}$. 


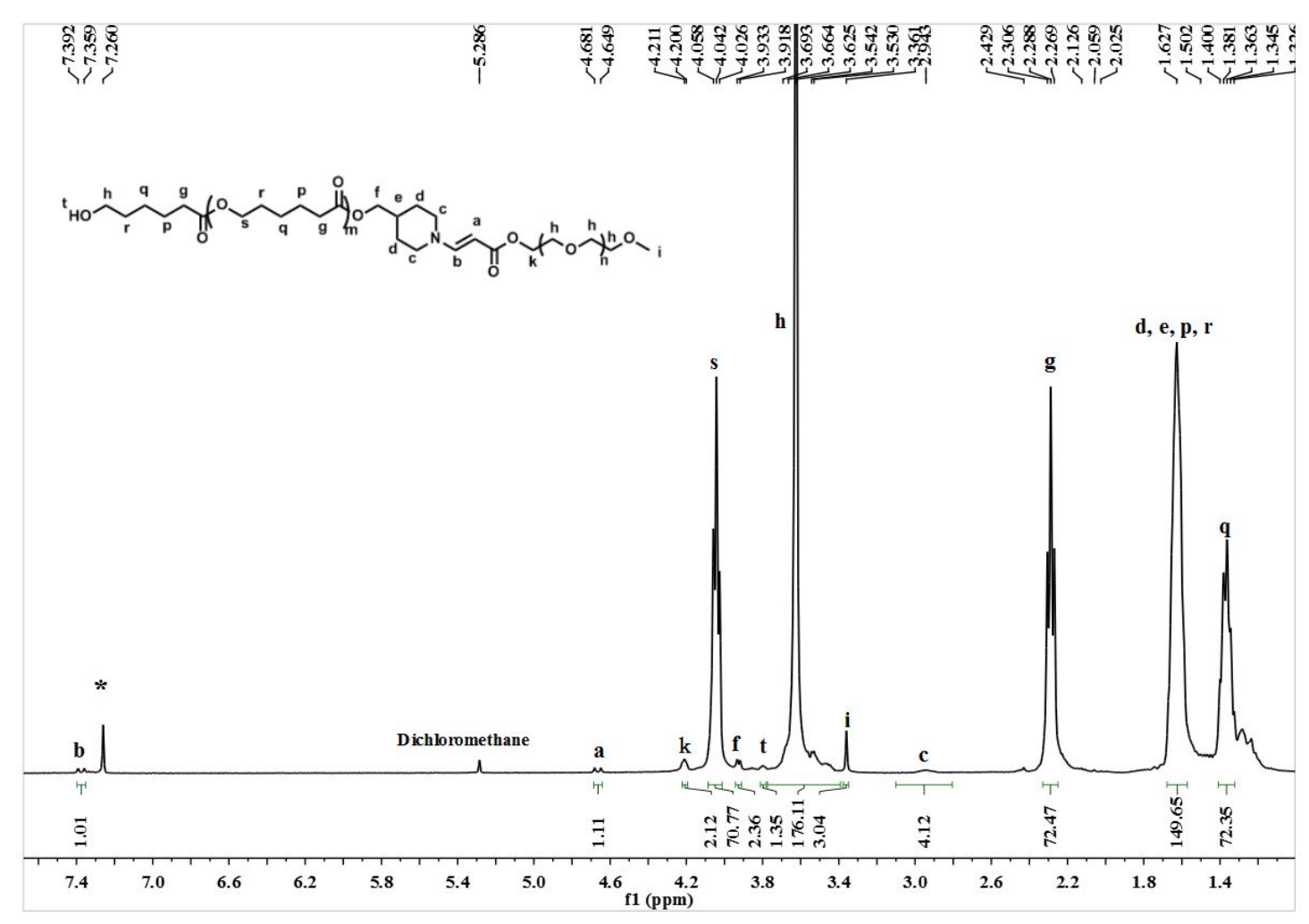

Figure S4. The ${ }^{1} \mathrm{H}$ NMR spectrum of SOR-mPEG- $b$-PCL in $\mathrm{CDCl}_{3}$.

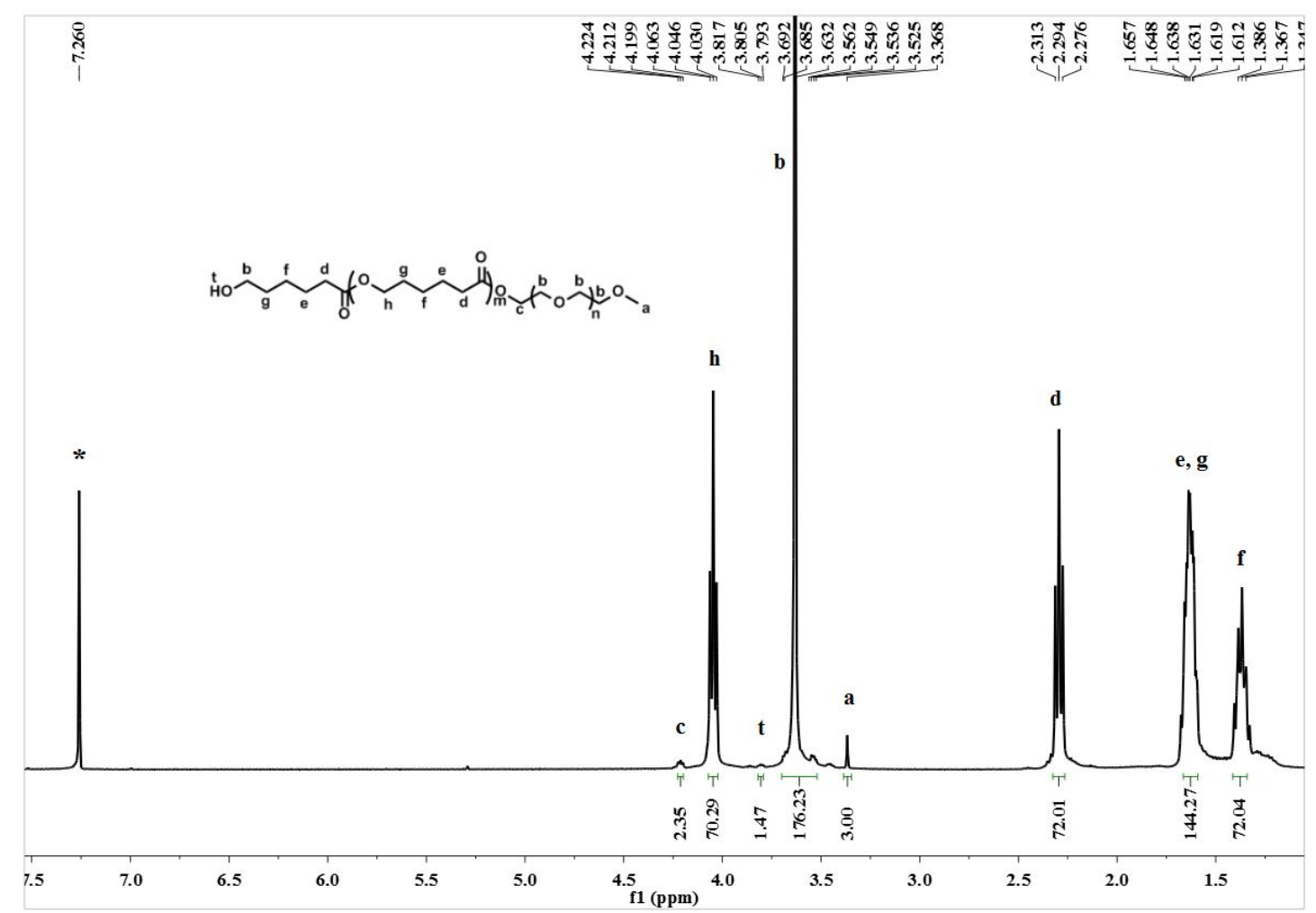

Figure S5. The ${ }^{1} \mathrm{H}$ NMR spectrum of mPEG- $b-\mathrm{PCL}$ in $\mathrm{CDCl}_{3}$. 


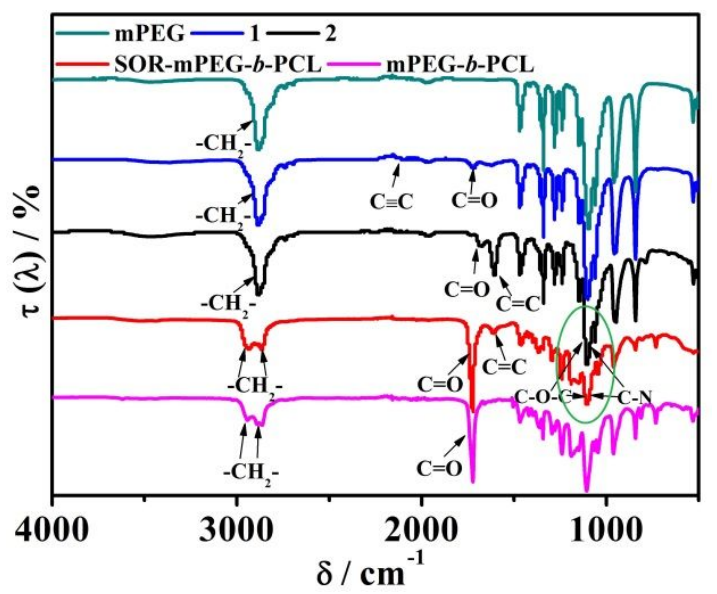

Figure S6. The Infrared spectra of mPEG, 1, 2, SOR-mPEG- $b$-PCL, mPEG- $b$-PCL.

Table S1. Characteristics of polymers

\begin{tabular}{|c|c|c|c|c|}
\hline polymer & $\begin{array}{c}\mathrm{M}_{\mathrm{n}}(\mathrm{NMR}) \\
\left(\mathrm{g} \mathrm{mol}^{-1}\right)\end{array}$ & $\begin{array}{c}M_{n}(G P C) \\
\left(\mathrm{g} \mathrm{mol}^{-1}\right)\end{array}$ & $\begin{array}{c}M_{w}(G P C) \\
\left(\mathrm{g} \mathrm{mol}^{-1}\right)\end{array}$ & $M_{w}(G P C) / M_{n}(G P C)$ \\
\hline SOR-mPEG $_{44}-b-\mathrm{PCL}_{35}$ & 6199 & 4655 & 6281 & 1.35 \\
\hline mPEG $_{44}-b-\mathrm{PCL}_{35}$ & 6032 & 4262 & 6532 & 1.53 \\
\hline
\end{tabular}

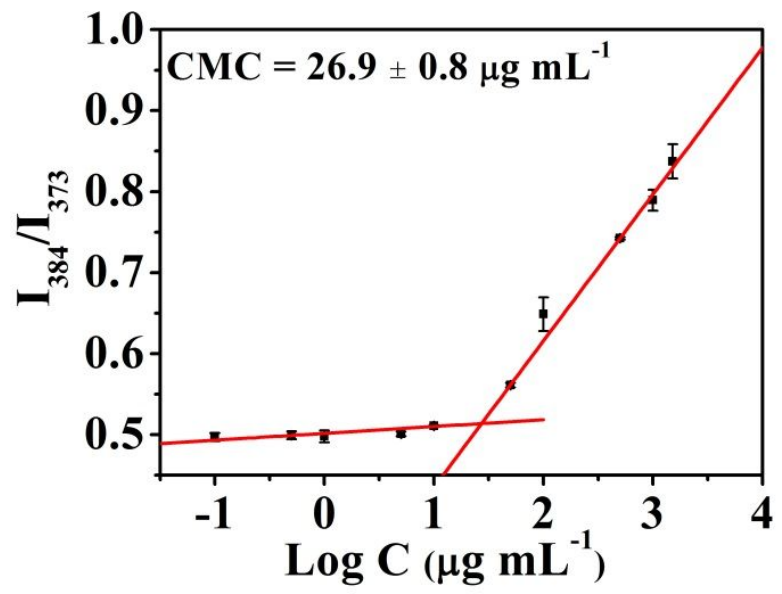

Figure S7. Critical micelle concentration of mPEG- $b$-PCL. 

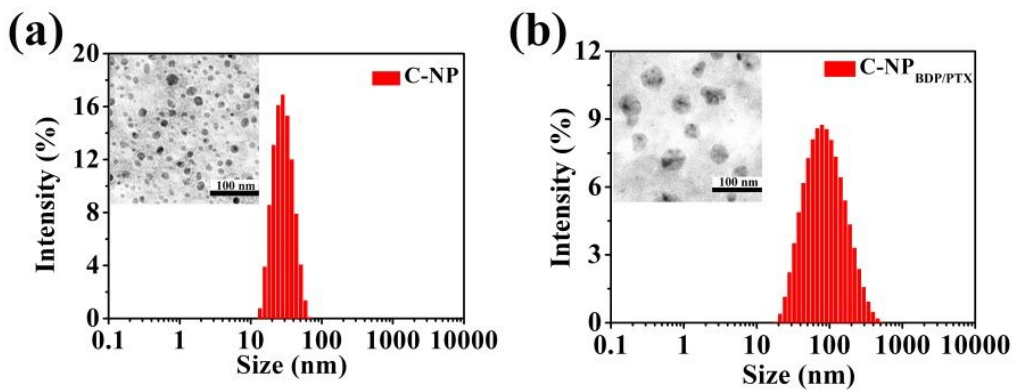

Figure S8. Dynamic light scattering and transmission electron microscope images (inset) of (a) C-NP and (b) $\mathrm{C}-\mathrm{NP}_{\mathrm{BDP} / \mathrm{PTX}}$.

Table S2. Physicochemical characteristics of BDP/PTX loaded nanoparticles.

\begin{tabular}{|c|c|c|c|c|c|c|c|c|}
\hline \multirow[t]{2}{*}{ Sample } & \multicolumn{2}{|c|}{$\begin{array}{c}\text { Feed Amount } \\
\text { (mg) }\end{array}$} & \multicolumn{2}{|c|}{$\begin{array}{c}\text { Loading Content } \\
\text { (wt\%) }\end{array}$} & \multicolumn{2}{|c|}{$\begin{array}{l}\text { Loading Efficiency } \\
\text { (wt\%) }\end{array}$} & \multirow[t]{2}{*}{$\begin{array}{l}\text { Size } \\
(\mathrm{nm})\end{array}$} & \multirow[t]{2}{*}{ PDI } \\
\hline & BDP & PTX & BDP & PTX & BDP & PTX & & \\
\hline SOR-NP & - & - & - & - & - & - & $52 \pm 21.7$ & 0.169 \\
\hline SOR-NP & 2 & 2 & $3.7 \pm 0.1$ & $2.5 \pm 0.1$ & $92.7 \pm 2.3$ & $62.7 \pm 2.8$ & $84 \pm 25.2$ & 0.210 \\
\hline C-NP & - & - & - & - & - & - & $28 \pm 9.0$ & 0.186 \\
\hline C-NP ${ }_{\text {BDP/PTX }}$ & 2 & 2 & $3.7 \pm 0.1$ & $2.6 \pm 0.1$ & $91.9 \pm 1.6$ & $64.4 \pm 1.8$ & $78 \pm 30.6$ & 0.215 \\
\hline
\end{tabular}

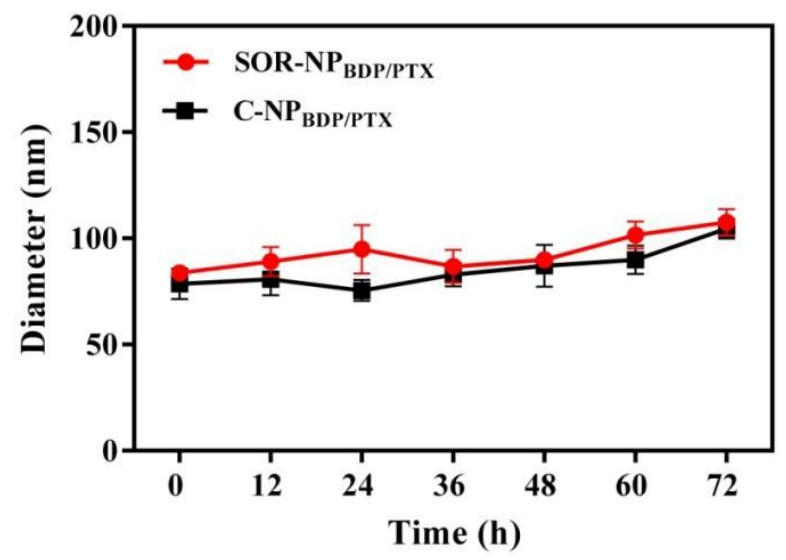

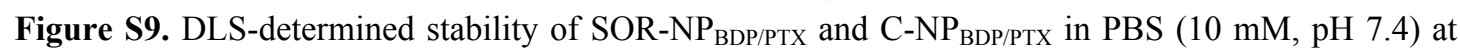
$37^{\circ} \mathrm{C}$. 
(a)

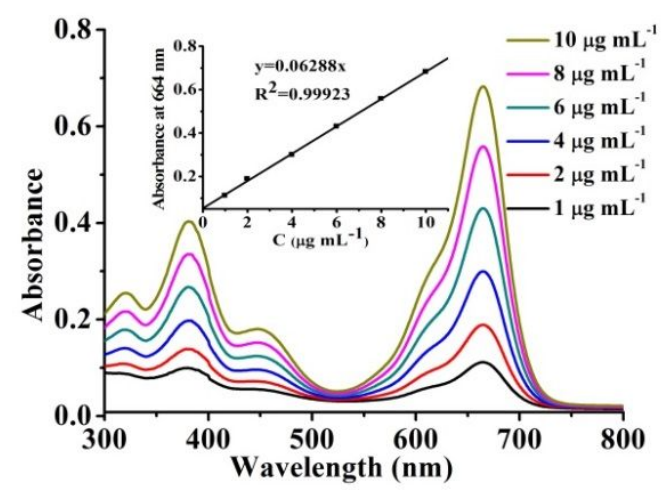

(b)

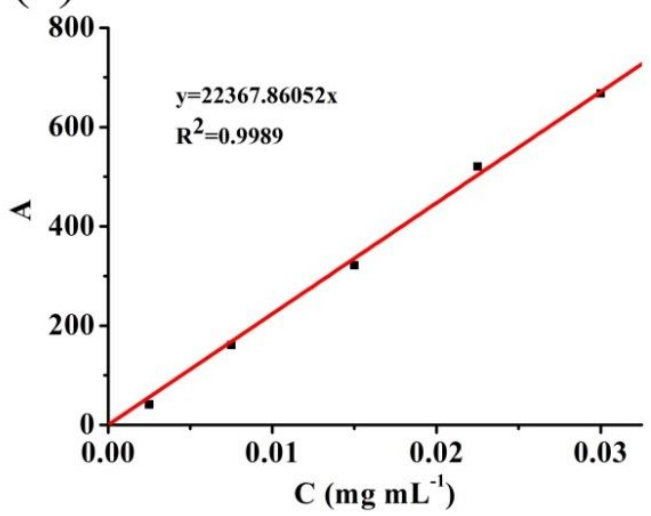

Figure S10. (a) UV-Vis absorption spectra of BDP at different concentrations in DMSO, (b) The integral area of HPLC peak versus the concentration of PTX.

(a)

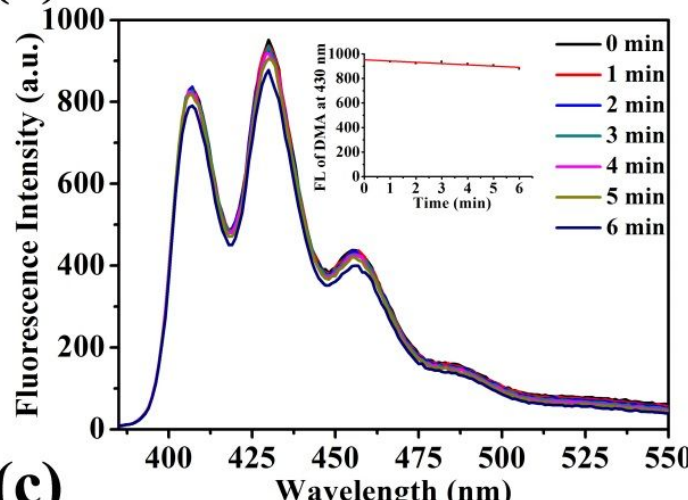

(c)

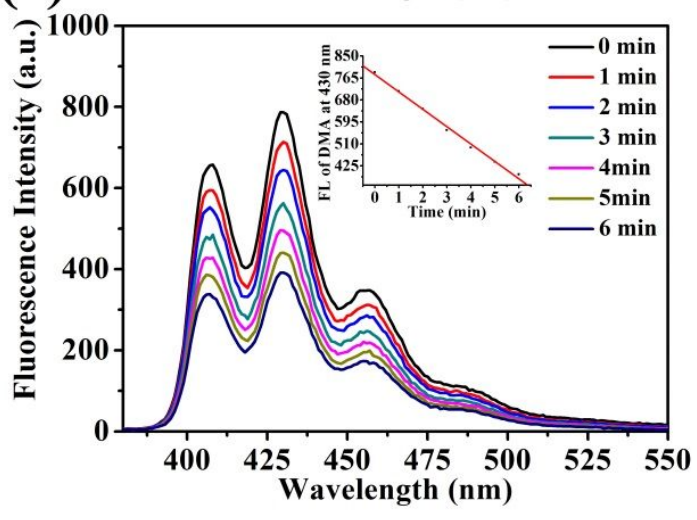

(b)
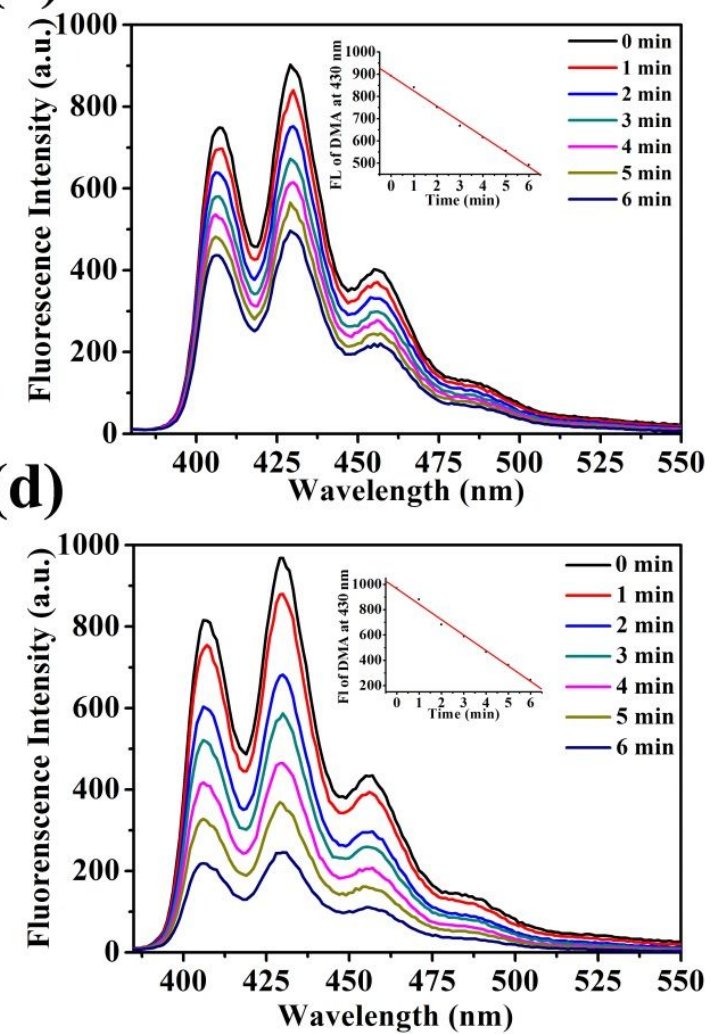

Figure S11. The fluorescence emission spectra of DMA at different durations of irradiation (660 nm, 5 $\mathrm{mW} \mathrm{\textrm {cm } ^ { 2 }}$ ) in the presence of (a) Free BDP in PBS, (b) C-NP $\mathrm{BDP} / \mathrm{PTX}_{\text {in PBS, (c) SOR-NP } \mathrm{BDP} / \mathrm{PTX}}$ in PBS, (d) Free BDP in DMSO. 


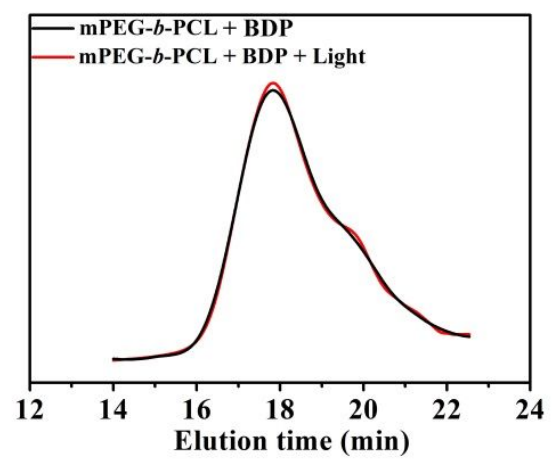

Figure S12. GPC profiles of mPEG- $b$-PCL with BDP $(\sim 5 \mathrm{wt} \%)$ under light irradiation $(660 \mathrm{~nm}, 5 \mathrm{~mW}$ $\mathrm{cm}^{-2}$ ) for $0.5 \mathrm{~h}$.

(a)

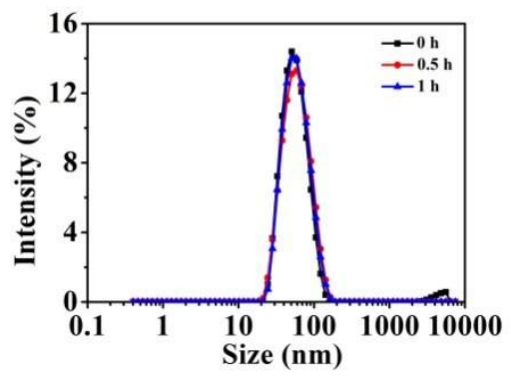

(b)

Without light With light

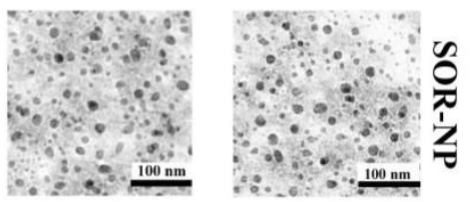

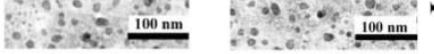

Figure S13. Average size change of (a) SOR-NP under various irradiation time $\left(660 \mathrm{~nm}, 5 \mathrm{~mW} \mathrm{~cm} \mathrm{~cm}^{-2}\right)$. (b) TEM images of SOR-NP without or with irradiation $\left(660 \mathrm{~nm}, 5 \mathrm{~mW} \mathrm{~cm} \mathrm{~cm}^{-2}, 1 \mathrm{~h}\right)$.

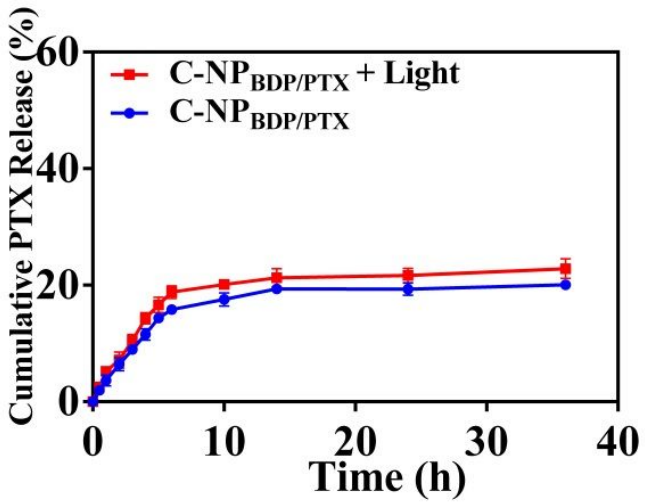

Figure S14. Cumulative PTX release profiles from C-NP ${ }_{\mathrm{BDP} / \mathrm{PTX}}$ without or with light irradiation (660 $\mathrm{nm}, 5 \mathrm{~mW} \mathrm{~cm}^{-2}$ ) for $1 \mathrm{~h}$. 

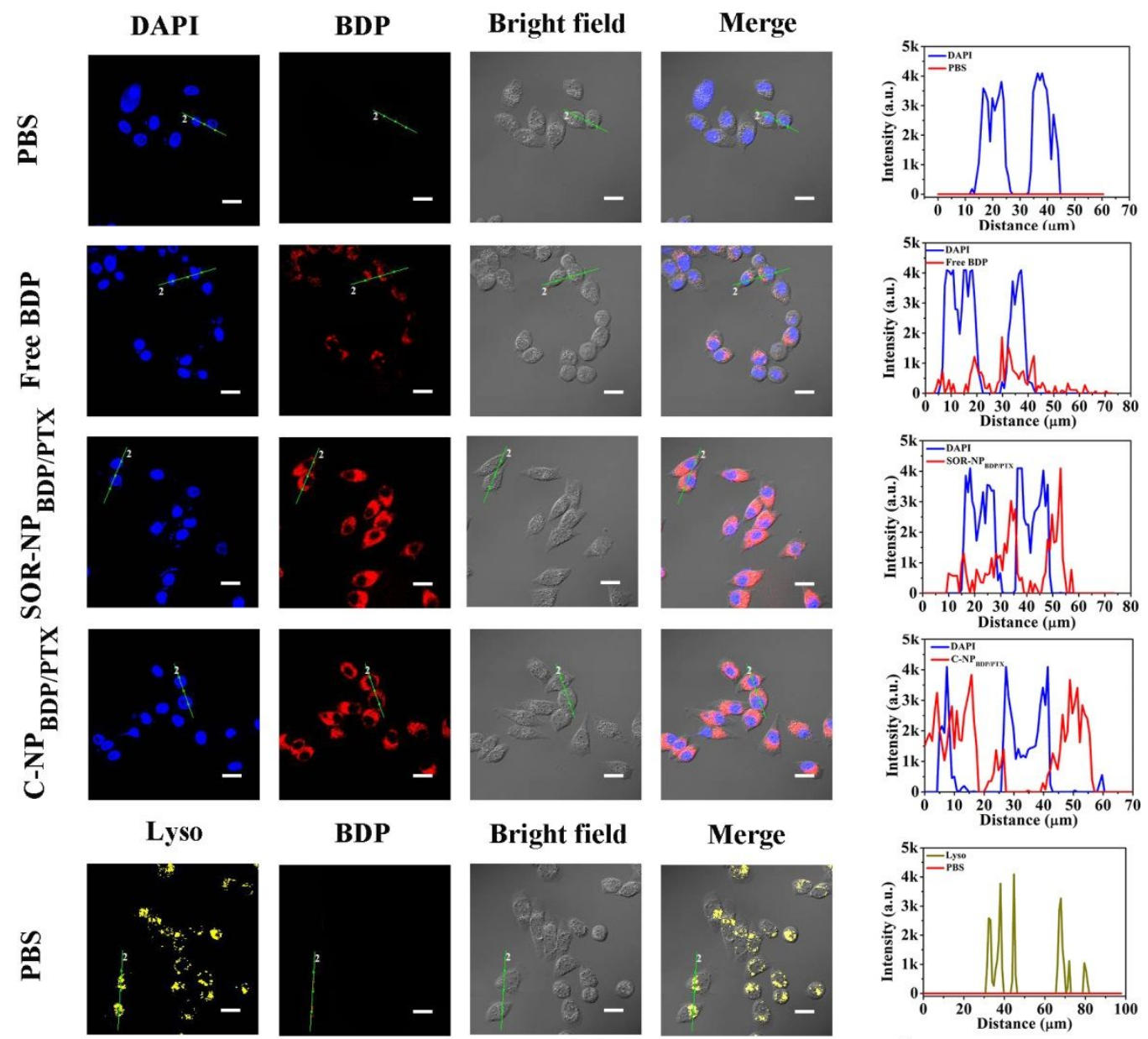

\section{Merge}
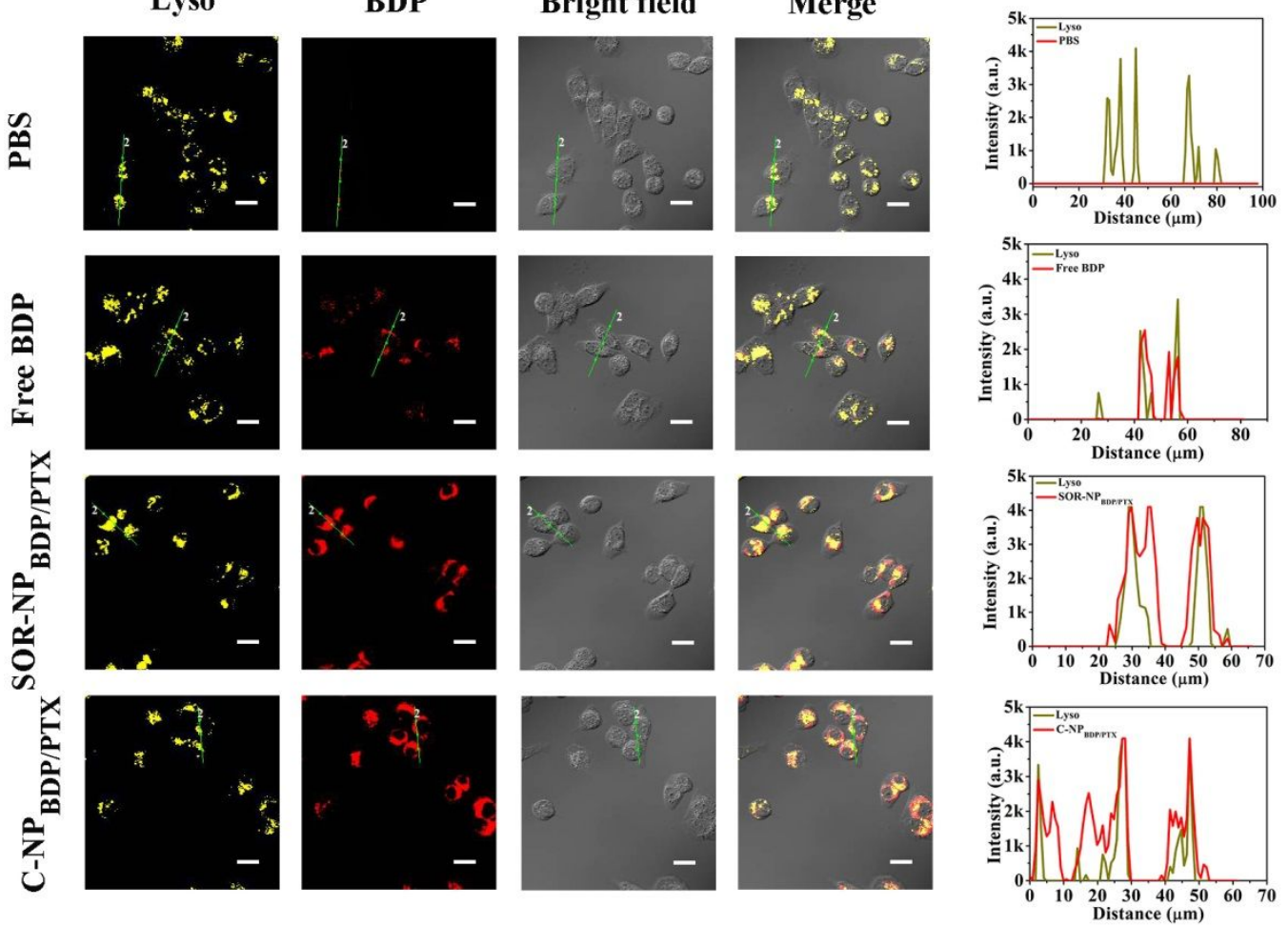

Figure S15. Cellular uptakes of SOR-NP $\mathrm{BDP}_{\mathrm{BPTX}}, \mathrm{C}-\mathrm{NP}_{\mathrm{BDP} / \mathrm{PTX}}$, Free BDP, and PBS in HepG2 cells, the nuclei and lysosomes of cells were stained with DAPI-trackers (blue) and lyso-trackers (yellow), respectively. The right-hand column shows the fluorescence profiles of tracker and formulation traced along the line. Scale bar: $20 \mu \mathrm{m}$. 

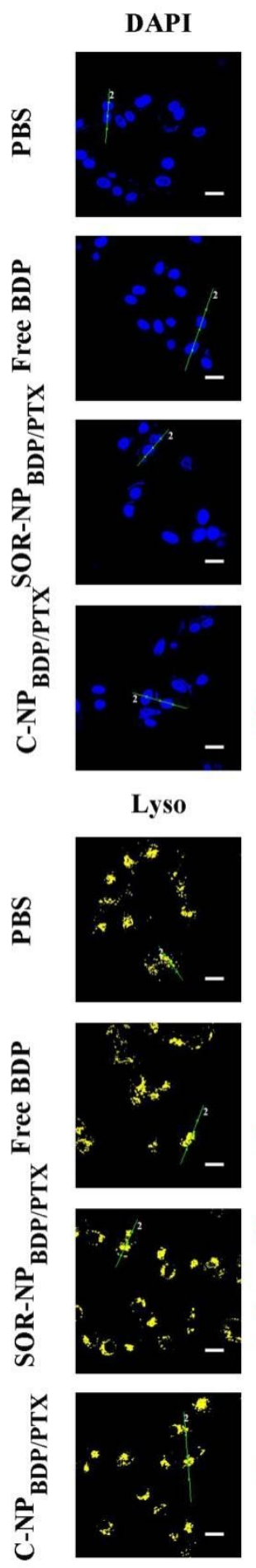

BDP
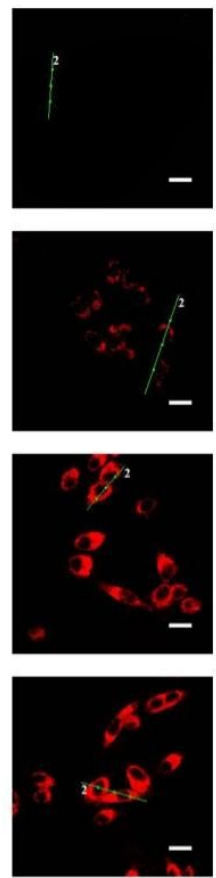

BDP
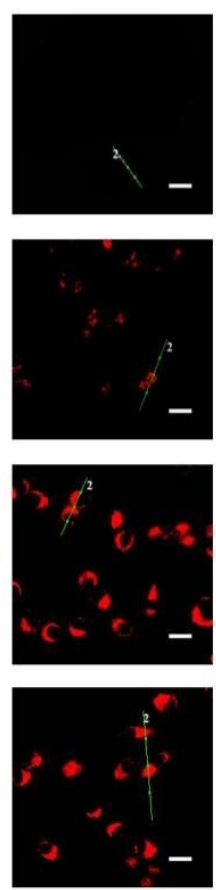
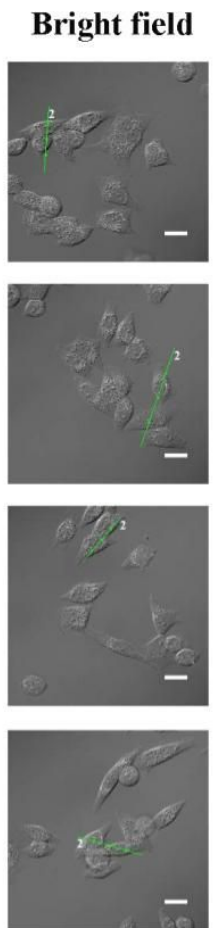

Bright field
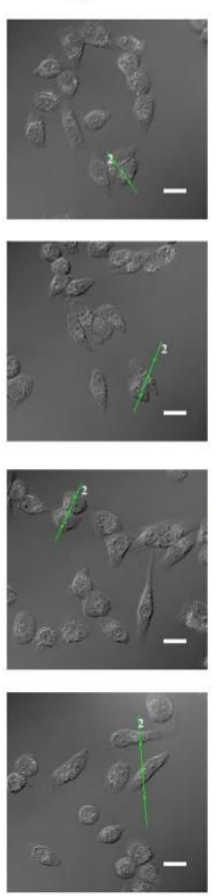
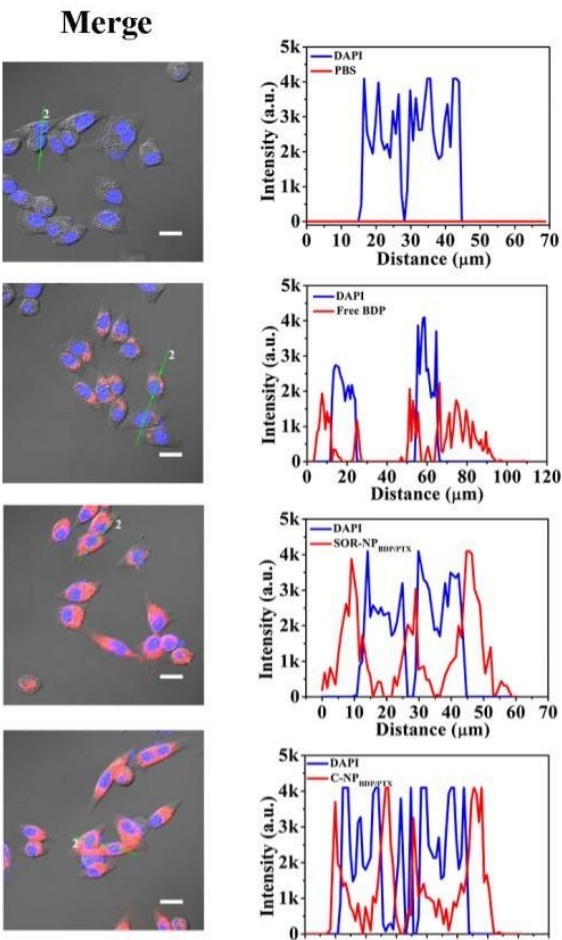

Merge
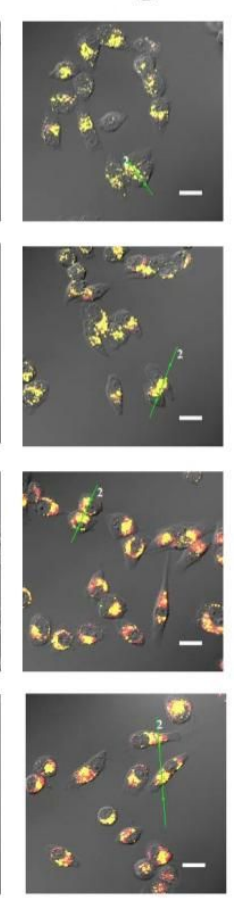
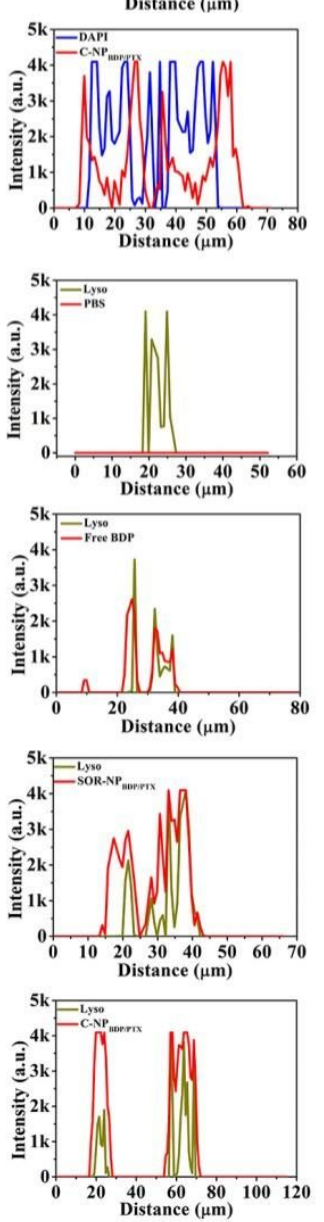

Figure S16. Cellular uptakes of SOR-NP $\mathrm{BDP} / \mathrm{PTX}_{\text {, }} \mathrm{C}-\mathrm{NP}_{\mathrm{BDP} / \mathrm{PTX}}$, Free BDP, and PBS in MCF-7 cells, the nuclei and lysosomes of cells were stained with DAPI-trackers (blue) and lyso-trackers (yellow), respectively. The right-hand column shows the fluorescence profiles of tracker and formulation traced along the line. Scale bar: $20 \mu \mathrm{m}$. 

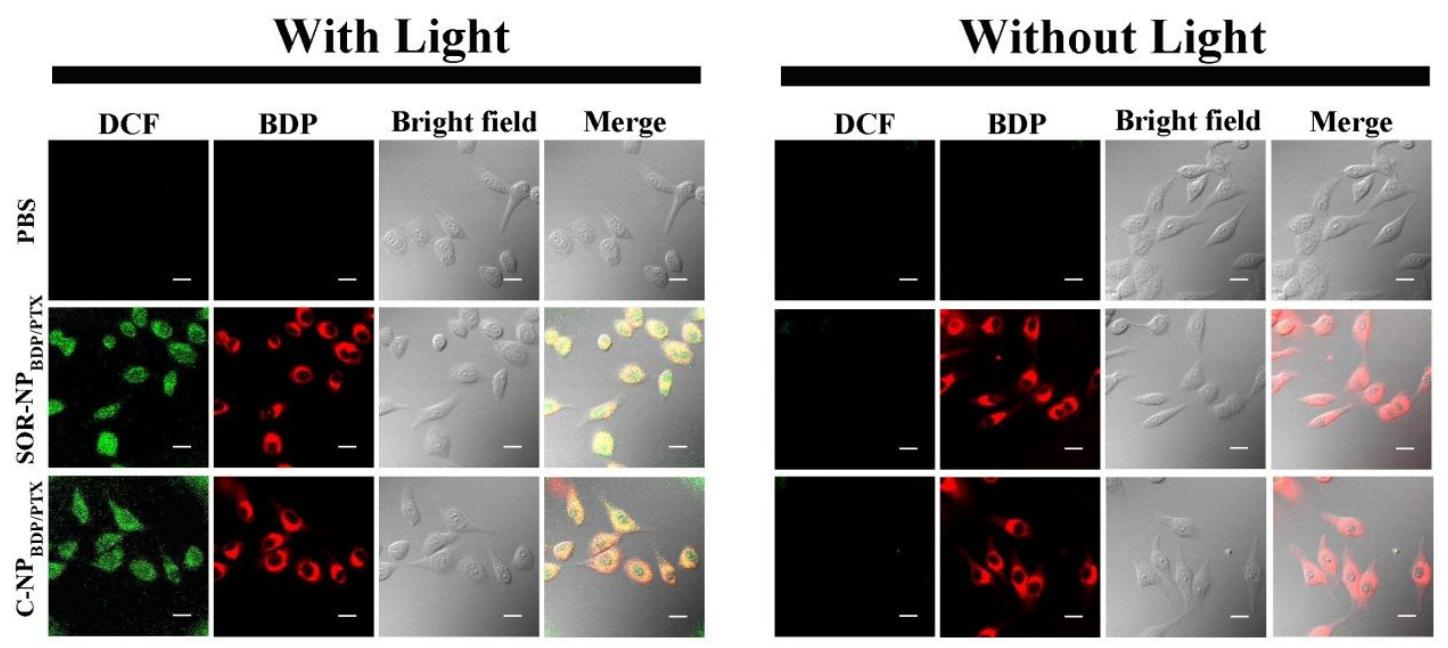

Figure S17. Intracellular ${ }^{1} \mathrm{O}_{2}$ production of SOR-NP $\mathrm{BDP} / \mathrm{PTX}$ and $\mathrm{C}-\mathrm{NP}_{\mathrm{BDP} / \mathrm{PTX}}$ without or with light irradiation (660 nm, $20 \mathrm{~mW} \mathrm{~cm}{ }^{-2}, 2 \mathrm{~min}$ ) in MCF-7 cells using DCFH-DA as the indicator. The green fluorescence is from DCF, the red fluorescence is from BDP. Scale bar: $20 \mu \mathrm{m}$.

(a)

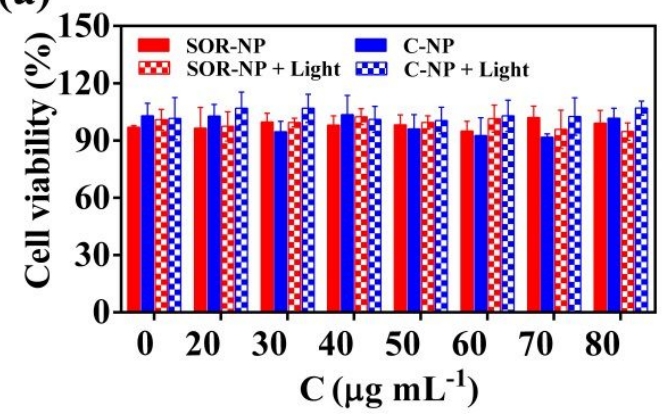

(b)

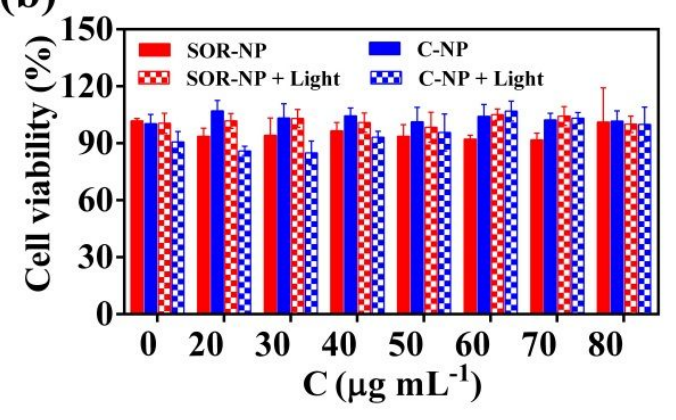

Figure S18. Cytotoxicity of SOR-NP and C-NP against (a) HepG2 and (b) MCF-7 cells in the absence/presence of light irradiation (660 nm, $\left.20 \mathrm{~mW} \mathrm{~cm}{ }^{-2}, 5 \mathrm{~min}\right)$. 


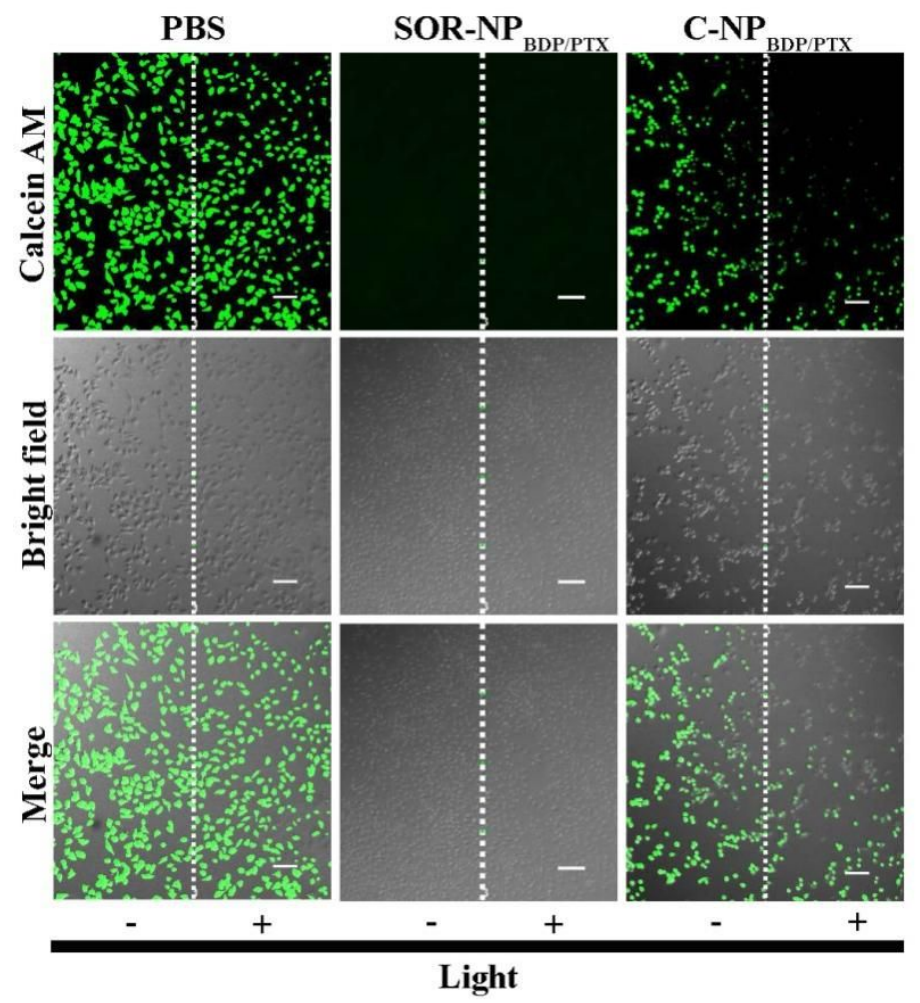

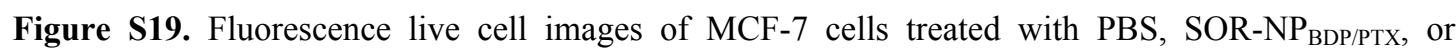
$\mathrm{C}-\mathrm{NP}_{\mathrm{BDP} / \mathrm{PTX}}$. Only half of each dish was irradiated with $660 \mathrm{~nm}$ laser $\left(20 \mathrm{~mW} \mathrm{~cm}{ }^{-2}, 5 \mathrm{~min}\right)$. Scale bar: $100 \mu \mathrm{m}$.

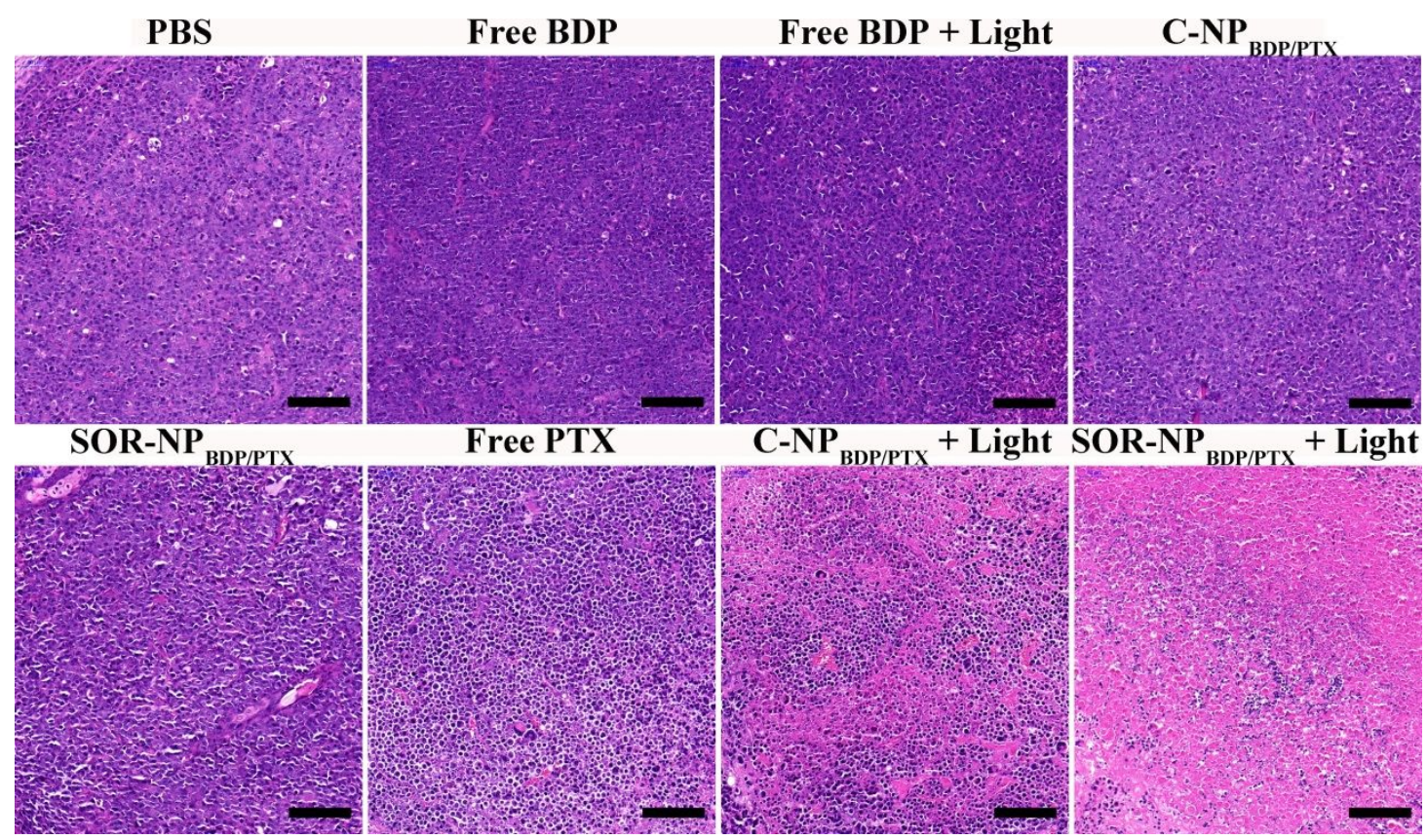

Figure S20. H\&E staining analysis of tumor tissues in different formulations (scale bar $=50 \mu \mathrm{m}$ ). 


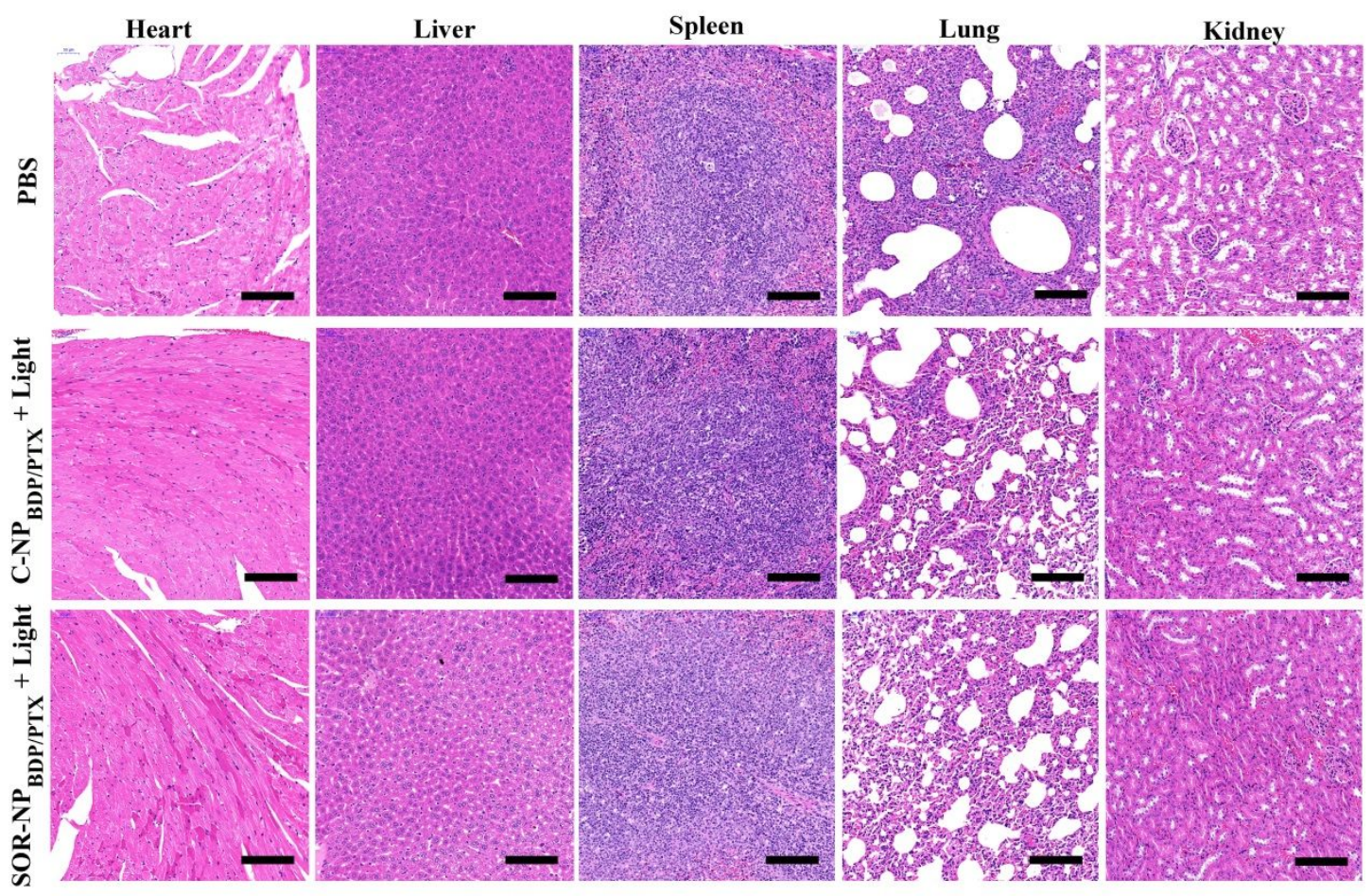

Figure S21. H\&E staining of heart, liver, spleen, lung and kidney (scale bar $=50 \mu \mathrm{m})$ 